CLINICAL STUDY

\title{
Testosterone substitution with a new transdermal, hydroalcoholic gel applied to scrotal or non-scrotal skin: a multicentre trial
}

\author{
B Kühnert, M Byrne, M Simoni, W Köpcke ${ }^{1}$, J Gerss ${ }^{2}$, G Lemmnitz ${ }^{3}$ and E Nieschlag for the Wolff Multi-Centre \\ Study Group \\ Institute of Reproductive Medicine, ${ }^{1}$ Department of Medical Informatics and Biomathematics, ${ }^{2}$ Coordinating Centre for Clinical Trials of the University. \\ Münster, Germany and ${ }^{3}$ Medical Department of Dr Wolff, Bielefeld, Germany \\ (Correspondence should be addressed to E Nieschlag, Institute of Reproductive Medicine of the University, Domagkstr. 11, D-48129 Münster, Germany; \\ Email: eberhard.nieschlag@ukmuenster.de)
}

\begin{abstract}
Objective: Testosterone-containing gels have improved testosterone substitution therapy, but they are associated with the risk of interpersonal transfer. Therefore, we tested a new hydroalcoholic $2.5 \%$ testosterone gel (TGW), which was removed by washing 10 min after administration.

Design: The gel was applied to scrotal or non-scrotal skin in comparison to two $2.5 \mathrm{mg}$ Androderm ${ }^{\circledR}$ patches in a randomised, three-arm, parallel-group, controlled multicentre trial over a period of 24 weeks. We included symptomatic hypogonadal men whose morning testosterone levels were $<10 \mathrm{nmol} / \mathrm{l}$. Either $1 \mathrm{~g}$ TGW was applied to scrotal skin $(n=54)$ or $5 \mathrm{~g}$ to non-scrotal skin $(n=56)$ once daily; the patch group $(n=52)$ applied two patches/day. Dose titration was allowed. Results: Whereas serum testosterone levels and the pre-post changes of the areas under the curve of testosterone and free testosterone between weeks 0 and 24 indicated equivalent treatment success for the patch and scrotal groups, the dermal gel group was significantly superior to the other two groups. Questionnaires on sexual function, mood and quality of life did not differ significantly between study groups, nor were prostate volume, prostate-specific antigen (PSA) levels and prostate symptoms different. However, tolerability was much better in the gel groups than the patch group.

Conclusion: Efficacy, safety and tolerability suggest TGW as a favourable treatment for hypogonadal patients.
\end{abstract}

European Journal of Endocrinology 153 317-326

\section{Introduction}

Testosterone gels have overcome most of the disadvantages of earlier modalities of testosterone substitution; that is, they induce physiological testosterone levels, skin reactions are rarely observed and short-term discontinuation of therapy is possible (1-3). The main cause for concern when using the gels is the possible interpersonal transfer of testosterone. Possible side effects of androgen transfer to women could be virilisation, while in children puberty might be induced prematurely. The necessity of dose titration affecting about $60 \%$ of patients is another shortcoming of the available gels (3).

To optimise androgen substitution therapy with gels, we tested a new hydroalcoholic testosterone gel (Wolff, Bielefeld, Germany), with the following differences to approved gels: 1 . the gel has a higher testosterone concentration $(2.5 \%) ; 2$. in addition to trunk skin we tested a new site of application, the scrotum, which is considered to have a fivefold higher resorption rate than other skin areas (4), so that the absolute amount of administered gel that is required is smaller; 3. we instructed patients to remove the gel $10 \mathrm{~min}$ after application, which is contrary to the protocol for the approved gels. We have compared this new gel $\left(\right.$ TGW $\sim$ Testocur ${ }^{\circledR}$ ) with a non-scrotal patch (Androderm ${ }^{\circledR} 2.5$, AstraZeneca, Wedel, Germany), the only transdermal application available when the study started in 2002, in a three-arm, parallel-group, open, randomised, controlled, multicentre, clinical trial over a period of 24 weeks. In preliminary studies, we have analysed the pharmacokinetics and interpersonal transfer of TGW in healthy, gonadotropin-suppressed men $(5,6)$, whereas this study evaluates efficacy, safety, tolerability and pharmacokinetic properties of TGW in hypogonadal patients during long-term treatment. Most of the patients suffered from primary or secondary hypogonadism; some had late-onset 
hypogonadism. The primary efficacy parameter was the pre-post changes of the serum testosterone levels between visits at the start and the end of the study on the basis of the area under the curve (AUC).

\section{Subjects and methods}

\section{Study design}

We enrolled 162 hypogonadal men in 14 university clinics, comprising urology, dermatology and endocrinology outpatient clinics and one urology practice, all located in Germany. All patients were of Caucasian origin except for one Asian and two patients of other ethnic origin. All patients gave written informed consent. The study was approved by the local Ethics Committees of all 15 study centres and performed according to Good Clinical Practice (7).

We included patients with primary, secondary or late-onset hypogonadism aged over 18 years with symptoms of testosterone deficiency and a testosterone level below $10 \mathrm{nmol} / \mathrm{l}$ (before $1100 \mathrm{~h}$ ). Patients who had been pretreated with testosterone underwent a washout period of 5 weeks before screening in the case of testosterone enanthate injections and 1 week in the case of oral or transdermal pretreatment. Exclusion criteria were severe acute or chronic illness, concomitant treatment with phenobarbital, relevant abnormalities in clinical chemistry, or haematology and body weight exceeding $\pm 20 \%$ of the normal body-mass index (BMI) $\left(16-30 \mathrm{~kg} / \mathrm{m}^{2}\right)$. Patients who met the inclusion criteria were assigned a randomisation number in ascending order. The randomisation took place according to the biometrician's internal randomisation SOP. A randomisation list was generated by block randomisation with a specified block size of six units. Block size was not quoted in the protocol and not revealed to the investigators. A randomisation protocol was kept for documentation.

Patients were assigned to one of three parallel arms: 1 . a dermal testosterone gel arm $(n=56), 2$. a scrotal testosterone gel arm $(n=54)$ and 3. a testosterone patch $\operatorname{arm}(n=52)$. The first group applied $5 \mathrm{~g}$ TGW, corresponding to $125 \mathrm{mg}$ testosterone, to an area of non-scrotal skin, as hairless as possible, between 0700 and $0900 \mathrm{~h}$. The gel was removed by washing $10 \mathrm{~min}$ after application. The dose could be decreased to $2.5 \mathrm{~g}$ per day or increased to 7.5 or $10 \mathrm{~g}$ per day after 4 or 8 weeks. The second group applied $1 \mathrm{~g}$ TGW, containing $25 \mathrm{mg}$ testosterone as active ingredient, to the scrotum between 0700 and $0900 \mathrm{~h}$. As with the first group, the gel had to be removed by washing $10 \mathrm{~min}$ after application. A dose adjustment was permitted at the discretion of the investigator after 4 or 8 weeks. The dose could be decreased to $1 \mathrm{~g} 1 \%$ TGW or increased to 2 or $2.5 \mathrm{~g} \mathrm{2.5 \%} \mathrm{TGW} \mathrm{per} \mathrm{day.}$

The third group comprised patients applying two patches of Androderm ${ }^{\circledR}$ at about $1000 \mathrm{~h}$ with a total daily testosterone release of $5 \mathrm{~g}$ per day. The testosterone transdermal patches (TTP) were applied to nonscrotal hairless skin. In this group, a dose adjustment was possible to one or three patches per day at weeks 4 and 8. The drug adjustments for individual patients within each group was based upon their testosterone levels and clinical symptoms.

Patients were assessed at 2-weekly and, from week 4 onward, at 4-weekly intervals over a period of 24 weeks. At each consultation, blood pressure, pulse rate, concomitant medication, adverse events, compliance and tolerability were checked. Skin reactions were assessed by subjective scoring. Compliance was judged according to the number of used/unused gel packages and patches. At every visit, patients had to answer three questionnaires: 1. the Derogatis Questionnaire on sexual function (sexual cognition/fantasy, sexual arousal, sexual behaviour/experience, sexual drive/relationship, orgasm), 2. the Mood Disorder Questionnaire involving 24 questions on good mood, alertness and calmness) and 3. the International Prostate Symptoms Score questionnaire (IPSS). Blood was collected in the morning not later than $1100 \mathrm{~h}$, about $2 \mathrm{~h}$ after gel application and $13 \mathrm{~h}$ after patch administration. Haematology, clinical chemistry and urine were analysed (at screening and at weeks 4, 12 and 24 ) in the local laboratories of the investigators, whereas serum for hormones, SHBG and prostatespecific antigen (PSA) determinations was stored at $-20{ }^{\circ} \mathrm{C}$ and transferred to the central laboratory (Institute of Reproductive Medicine of the University, Münster, Germany).

Testosterone levels were measured during the course and at the end of the study. For testosterone kinetics, performed at weeks 0, 4, 12 and 24, patients were assessed at 2-hourly intervals between 0800 and $1800 \mathrm{~h}$ and, optionally (except week 0 , when it was obligatory), at $0800 \mathrm{~h}$ the following morning. Parallel to testosterone, dihydrotestosterone (DHT) levels were also measured along with kinetics in the scrotal group, whereas in the patch and the dermal group DHT levels were measured only once a day at time point 0 ; that is, just before the gel application or $13 \mathrm{~h}$ after patch application. In addition, DHT was measured $2 \mathrm{~h}$ after gel application in the dermal group on the kinetic day of week 24 , so that the levels could be compared with preceding hormone analyses on non-kinetic days. When combined kinetics of testosterone and DHT were calculated, the 8 o'clock DHT levels (time point 0 of the kinetics) were added to the testosterone levels in the dermal and the patch groups.

Prostate volume was assessed by transrectal ultrasonography.

\section{Hormone determinations}

LH, FSH, SHBG and PSA were determined by solid phase two-site fluoroimmunometric assay (IFMA), 
testosterone (T) by an enzyme immunoassay (EIA), estradiol by solid-phase fluoroimmunoassay (FIA), and DHT by a radioimmunoassay (RIA) with extraction and an oxidation step. Further assay characteristics are provided in Table 1.

\section{Statistical analysis}

Statistical analysis was performed according to the 'intention-to-treat-principle' (ITT); that is, all randomised patients who did not drop out before week 12 were analysed. Additionally, 'per protocol analysis' (PP) was performed and included all patients of the intention-to-treat-population without major deviations to the protocol. We created two different PP-populations (Fig. 1).

The basic variables of the randomised groups were checked for equal distribution with the KruskalWallis test for continuous variables and for qualitative variables with the Fisher or chi-square test.

The primary efficacy parameter was the pre-post changes of the serum $\mathrm{T}$ level between week 0 and week 24 on the basis of the area under the curve (AUC). AUC was calculated at the kinetic days by the linear trapezoid method. An ANOVA F-test was performed to test for differences in (log-transformed) means between any of two treatment groups, and $95 \%$ simultaneous confidence intervals of log-transformed treatment contrasts between two treatment groups were calculated by the Scheffe method. Standardised confidence intervals were obtained by dividing 95\% simultaneous confidence intervals by the estimated standard error of the respective parameter. If the standardised confidence interval between two treatments were within the range of equivalence $[-0.7$, 0.7], both treatments were considered equivalent. Additionally, the $P$ value was calculated. The average testosterone concentration $\left(\mathrm{C}_{\mathrm{ave}}\right)$ corresponds to the AUC divided by the time interval between the first and the last testosterone measurement. Efficacy parameters were assumed to be of log normal distribution. The log-transformed values were evaluated by a general linear model. All statistical tests were two-sided with an alpha-level of 0.05 . When results were presented by box plots, they were created by the following procedure: the box includes data from the 25th to the 75 th percentiles; consequently, it contains the central $50 \%$ of data. The median of all data is marked within the box with a bar. Whiskers are drawn to the nearest value not beyond a standard span from the quartiles, the standard span being defined as 1.5 times (interquartile range). Points beyond the whiskers (outliers) are drawn individually.

Statistics were performed by the statistical software S-PLUS, Version 6.1 (Insightful Corp. Seattle, WA, USA) and SAS, Version 8.2 (SAS Institute, Cary, NC, USA).

\section{Results}

\section{Patient populations}

The flow of patients through the study is shown in Fig.1. Baseline characteristics (group size, age, weight, height and BMI) were not significantly different between the three groups. The percentage of pretreated patients was higher in the gel groups than the patch group (Table 2). 72 of all randomised patients had primary hypogonadism, 45 had secondary hypogonadism and 56 patients were characterised as ageing men with late-onset hypogonadism. Eleven patients had lateonset hypogonadism combined with primary hypogonadism. Compliance was high in all three treatment groups with a mean of $94.9 \%$ and without significant differences between the groups.

\section{Drug adjustment}

Dose adjustment resulted in a decreased dose of one patch/day and in an increased dose of 3 patches/day in six patients, respectively from week 8 onward in the TTP group, so that $76.9 \%$ of patients maintained their original dose of two patches per day.

In the scrotal group, $96.2 \%$ maintained their dose of $1 \mathrm{~g} /$ day and the dose was increased to $2 \mathrm{~g} /$ day or $2.5 \mathrm{~g} /$ day at week 4 in one patient respectively.

In the dermal group, dose adjustment resulted in a decreased dose of $2.5 \mathrm{~g} / \mathrm{d}$ in three patients $(5.3 \%)$ and in an increased dose of 7.5 or $10 \mathrm{~g}$ /day in $5(8.9 \%)$ and seven $(12.5 \%)$ patients, respectively, from week 8 onward.

Table 1 Characteristics of the assays used in the study.

\begin{tabular}{|c|c|c|c|}
\hline Assay (normal male reference range) & $\begin{array}{l}\text { Least detectable } \\
\text { concentration }\end{array}$ & $\begin{array}{c}\text { Within-assay } \\
\text { variability (\%) }\end{array}$ & $\begin{array}{c}\text { Between-assay } \\
\text { variability (\%) }\end{array}$ \\
\hline Testosterone $(>12 \mathrm{nmol} / \mathrm{l})$ & 0.69 & 4.2 & 12.5 \\
\hline $\mathrm{LH}(2-10 \mathrm{U} / \mathrm{I})$ & 0.13 & 1.5 & 8.2 \\
\hline $\mathrm{FSH}(1-7 \mathrm{U} / \mathrm{I})$ & 0.245 & 1.8 & 4.5 \\
\hline Estradiol (<250 pmol/l) & 12.5 & 3.9 & 6.0 \\
\hline SHBG (11-71 nmol/l) & 6.25 & 2.8 & 7.8 \\
\hline PSA $(<4 \mu \mathrm{g} / \mathrm{l})$ & 0.199 & 1.4 & 4.8 \\
\hline
\end{tabular}




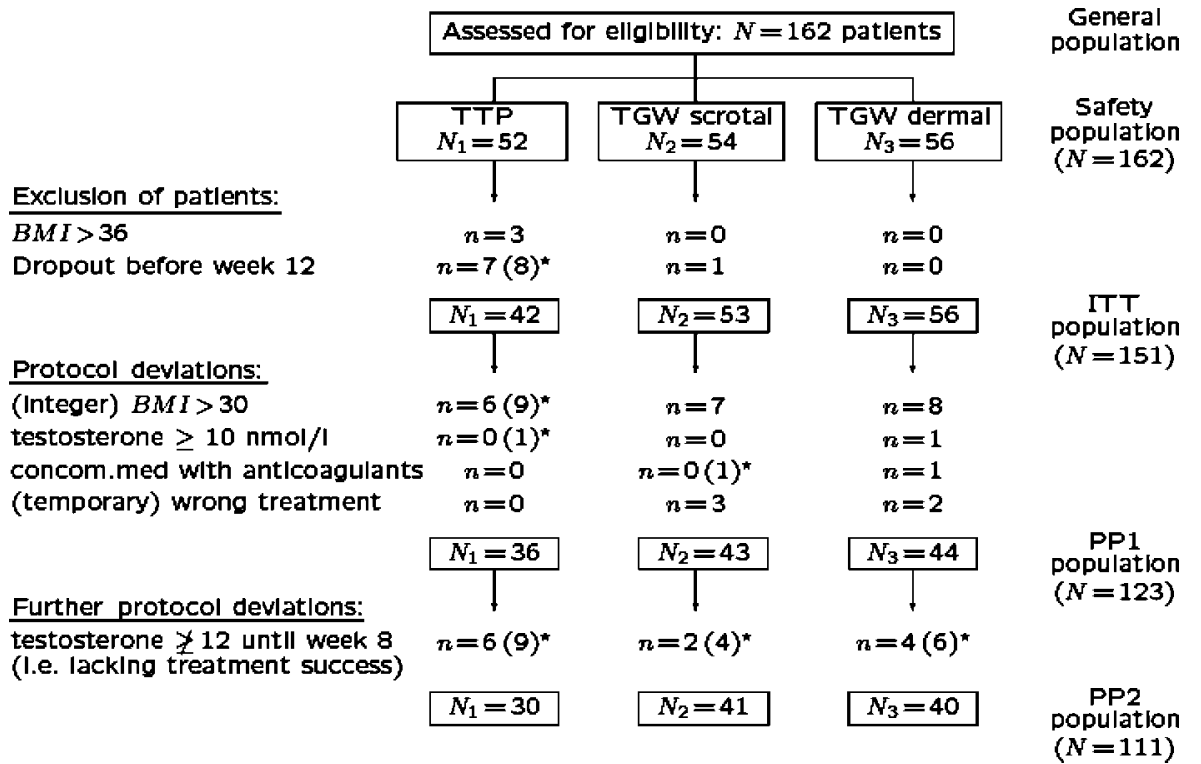

Figure 1 Disposition of patients. *Values in parentheses show number of patients to be excluded, disregarding previous exclusions in the flowchart.

\section{Testosterone/free testosterone}

Testosterone kinetics at weeks 0, 12 and 24 are presented in Fig. 2. Similar results were seen for free testosterone at week 24 (data not shown). Two outliers (in the dermal and the patch group), who were already in the eugonadal range at the second morning of the first kinetic day (week 0 ), were probably related to premature application of the study drug. Median testosterone levels during the course of the study are provided in Fig. 3A.

The change of the $\mathrm{C}_{\mathrm{ave}}$ of testosterone and free testosterone between weeks 0 and 24 was significant in all three groups and revealed equivalent treatment success for the patch and scrotal gel groups, whereas the dermal gel group was significantly superior to the other two groups. This was true for the ITT as well as for the PP analysis, and the results were confirmed by the Scheffe method and the test of equivalence. One exception was found in the PP2 analysis of free testosterone, which showed lower treatment success for the scrotal group compared with the patch in the test of equivalence. However, the Scheffe test did not show a significant difference between both groups. Further pharmacokinetic parameters are listed in Table 3.

Table 2 Baseline characteristics of the study groups (ITT population). Results are presented as median \pm IQ-range.

\begin{tabular}{lccc}
\hline Parameter & Patch & Scrotal & Dermal \\
\hline Count & 42 & 53 & 56 \\
Age (years) & $53 \pm 16$ & $50 \pm 21$ & $52.5 \pm 22.5$ \\
Height $(\mathrm{cm})$ & $177.5 \pm 13.5$ & $180 \pm 7$ & $179.5 \pm 10.3$ \\
Weight $(\mathrm{kg})$ & $83 \pm 17.25$ & $90 \pm 17$ & $85 \pm 18.3$ \\
BMI $\left(\mathrm{kg} / \mathrm{m}^{2}\right)$ & $26.2 \pm 2.9$ & $28.4 \pm 4.1$ & $26.2 \pm 5.2$ \\
Pretreatment with & 55 & 71.4 & 87 \\
$\quad$ testosterone $(\%)$ & & & \\
$(P=0.003)$ & & & \\
\hline
\end{tabular}

\section{DHT}

Median DHT levels (nmol/l \pm IQ range), measured $2 \mathrm{~h}$ after gel application, are provided in Fig. 4. Median DHT levels at week 24 before gel application increased 6.25 times in the scrotal group $(2.5 \pm 4.4)$ and 5 times

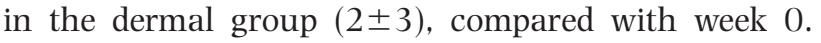
Looking at the combined levels of testosterone and DHT, the kinetics at week 24 demonstrate that the patch and scrotal gel groups also matched the eugonadal range. The combined testosterone and DHT levels during the course of the study are provided in Fig. 3B. Furthermore, the change of the $C_{\text {ave }}$ of the combined measurement of testosterone and DHT between weeks 0 and 24 revealed treatment success for scrotal and dermal gel groups significantly superior to the patch group and for the dermal group against the scrotal gel group, when the test of equivalence was used. However, the Scheffe method detected a significantly better treatment effect for only the dermal gel group compared with the patch group. Again, results were identical in the ITT and the PP analysis. Pharmacokinetic parameters of DHT in the scrotal group are summarised in Table 3.

\section{Estradiol}

Estradiol levels increased between weeks 0 and 4 and then remained within the normal range throughout the study without significant differences between groups (Fig. 5). SHGB (sex hormone binding globulin) did not change during the study in any of the groups.

\section{Gonadotropins}

Luteinising hormone (LH) and follicle-stimulating hormone (FSH) levels were suppressed in conjunction 


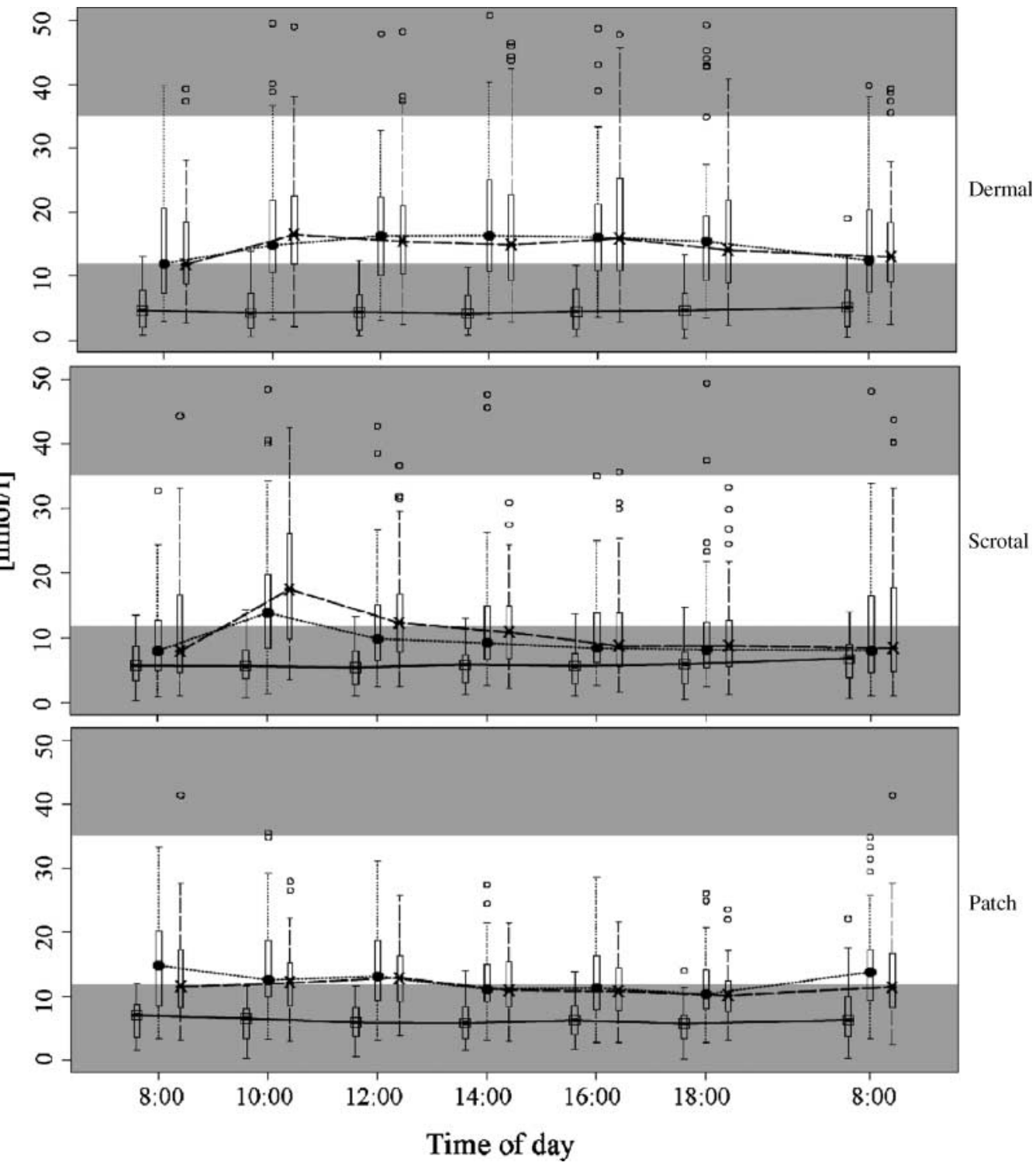

Figure 2 Median testosterone kinetics (ITT) in the dermal, scrotal and the patch groups at weeks $0(-\square-), 12(-\bullet)$ and $24(-\star)$.

with therapy without significant differences between the groups (Fig. 6).

\section{Prostate volumes and PSA levels}

Prostate volume did not show significant differences between the groups and remained constant throughout the study. PSA levels were slightly augmented between weeks 0 and 4 and then stayed consistently within the normal range. One patient from the scrotal group had slightly elevated PSA levels $(4.6 \mu \mathrm{g} / \mathrm{l})$ at the beginning of the study, which increased to $4.9 \mu \mathrm{g} / \mathrm{l}$ at the end of the study. Digital rectal examination and ultrasound of the patient did not indicate prostate cancer at patient follow-up. PSA levels were $4 \mu \mathrm{g} / \mathrm{l}$. Prostate volumes and PSA levels are shown in Fig. 7.

\section{Haematology, clinical chemistry and urinalysis}

All haematological parameters remained within the normal range throughout the study without differences between groups and without significant trends. However, when we analysed those patients who had not been pretreated with testosterone, haemoglobin, haematocrit and erythrocytes increased significantly within the normal range in all treatment groups. These parameters remained constant in patients of the scrotal and dermal gel groups who had been pretreated, whereas pretreated patients of the patch group revealed a significant decrease in haemoglobin levels within the normal range. All median levels of clinical chemistry and urinalysis remained within 


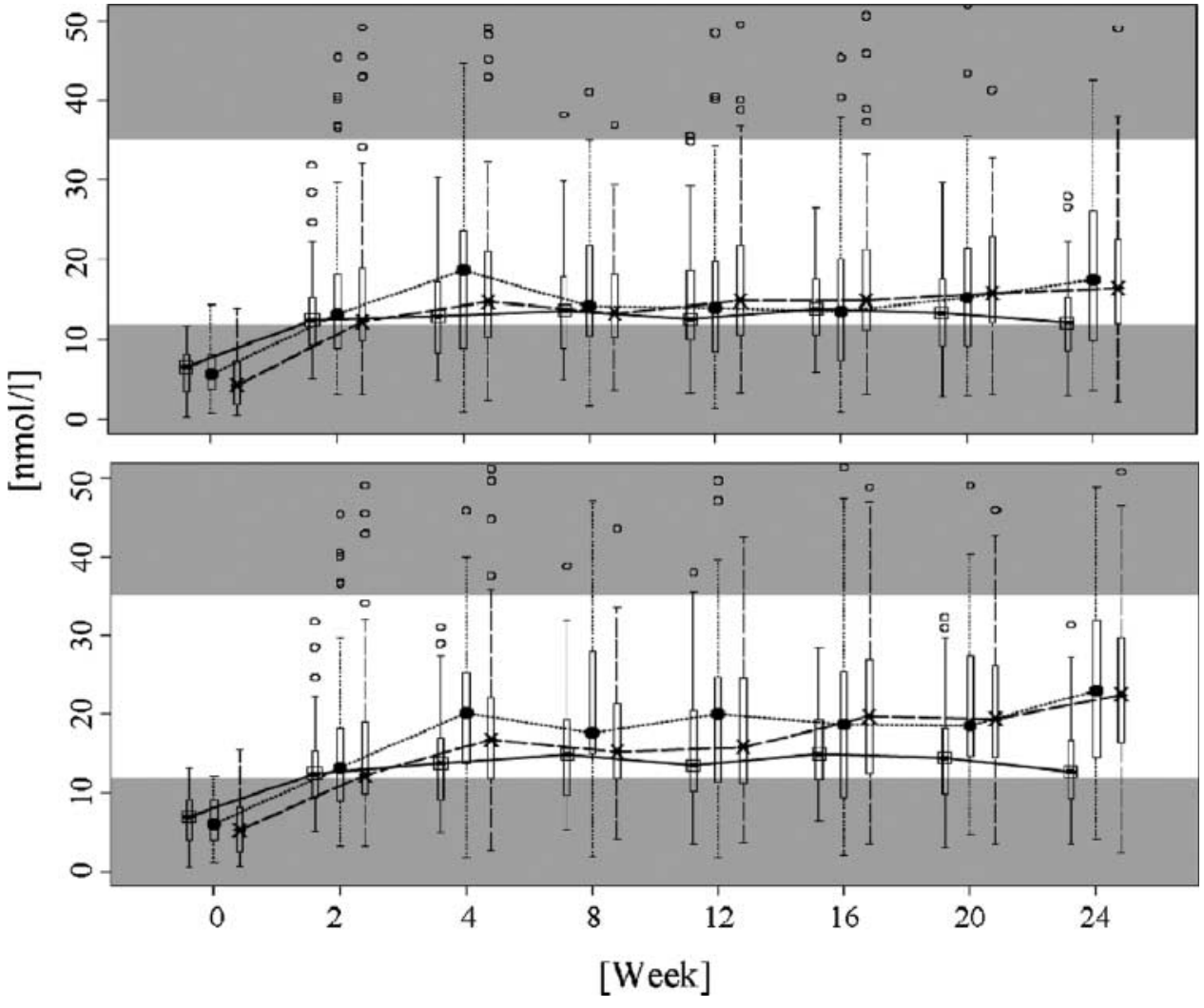

Figure 3 Median testosterone levels $(A)$ and median testosterone ( $\mathrm{T}$ ) plus DHT levels (ITT) (B), measured at $1000 \mathrm{~h}$, at weeks $0-24$ in the dermal $(-\square-)$, scrotal $(\rightarrow-)$ and patch groups $(-x)$.

Table 3 Pharmacokinetic parameters of T, free T and DHT at week 24 (Median \pm IQ-range). Parameters of the patch group are given in italics, as the patch was applied about 10 hours before the start of the kinetics.

\begin{tabular}{|c|c|c|c|c|}
\hline & $\mathrm{C}_{\text {ave }}$ & $C_{\min }$ & $C_{\max }$ & $t_{\max }$ \\
\hline Testosterone & $(\mathrm{nmol} / \mathrm{l})$ & $(\mathrm{nmol} / \mathrm{l})$ & $(\mathrm{nmol} / \mathrm{l})$ & (h) \\
\hline Patch & $11.0 \pm 4.3$ & $8.1 \pm 4.3$ & $15.7 \pm 6.9$ & $15 \pm 9.1$ \\
\hline Scrotal gel & $10.46 \pm 7.9$ & $5.86 \pm 5.0$ & $19.71 \pm 17.0$ & $2.5 \pm 23.2$ \\
\hline Dermal gel & $14.76 \pm 10.1$ & $9.34 \pm 6.3$ & $21.28 \pm 18.6$ & $8 \pm 6.0$ \\
\hline Free testosterone & $(\mathrm{pmol} / \mathrm{l})$ & $(\mathrm{pmol} / \mathrm{l})$ & $(\mathrm{pmol} / \mathrm{l})$ & (h) \\
\hline Patch & $284.8 \pm 120.9$ & $178.7 \pm 94.8$ & $379.1 \pm 185.1$ & $14.5 \pm 18.7$ \\
\hline Scrotal gel & $253.4 \pm 160.3$ & $110.2 \pm 114.4$ & $467.9 \pm 415.7$ & $2.5 \pm 23.2$ \\
\hline Dermal gel & $312.7 \pm 369.7$ & $189.2 \pm 147.3$ & $442.4 \pm 676.8$ & $8 \pm 5.9$ \\
\hline DHT & $(\mathrm{nmol} / \mathrm{l})$ & $(\mathrm{nmol} / \mathrm{l})$ & $(\mathrm{nmol} / \mathrm{l})$ & (h) \\
\hline Scrotal gel & $3.9 \pm 2.9$ & $1.4 \pm 2.8$ & $6.4 \pm 3$ & $2.4 \pm 6.1$ \\
\hline
\end{tabular}
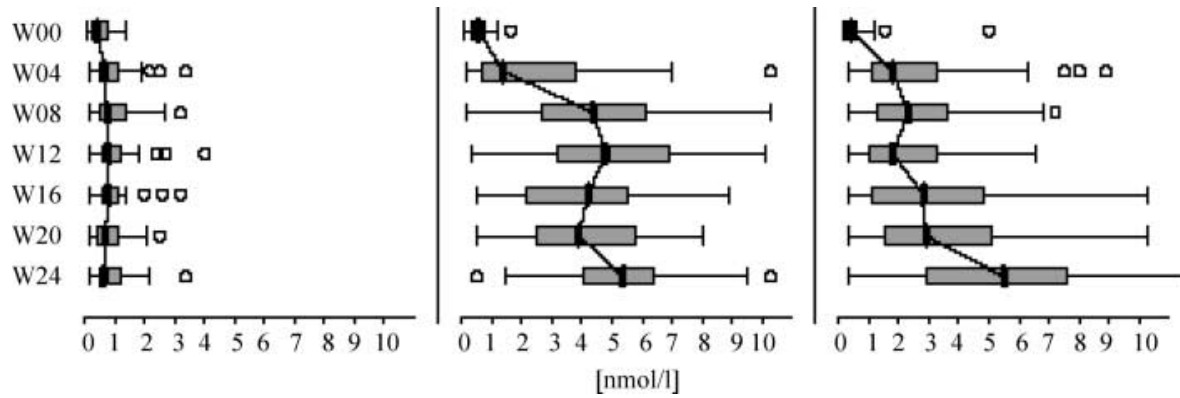

Figure 4 Median DHT levels in the patch, scrotal and dermal groups at weeks 0-24 (W00-24). At weeks 4 and 12, DHT levels were measured before gel application in the dermal group; otherwise, they were determined $2 \mathrm{~h}$ after gel application or $8-10 \mathrm{~h}$ after patch application. 


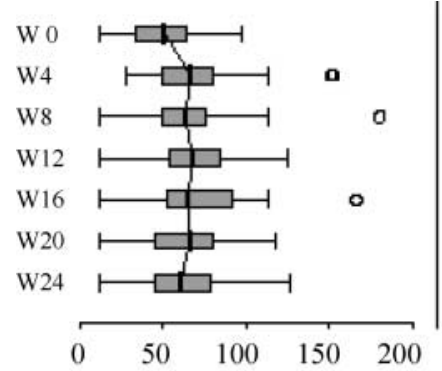

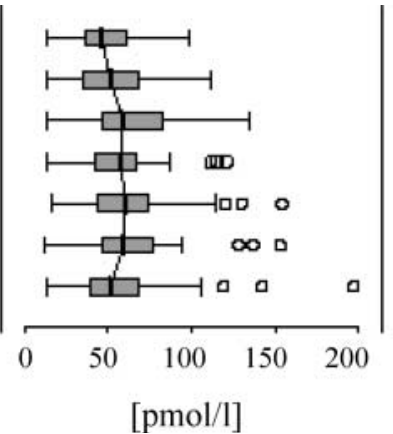

$[\mathrm{pmol} / \mathrm{l}]$

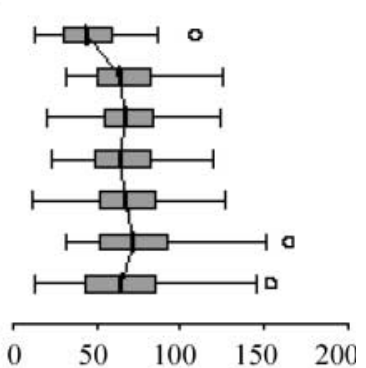

$100 \quad 150 \quad 200$
Figure 5 Estradiol levels in the patch, scrotal and dermal groups at weeks 0-24 (W00-24). normal ranges and were not significantly different between the three treatment groups.

\section{Questionnaires}

The patch and the dermal gel groups showed a statistically significant improvement in all three categories of the Mood Disorder Questionnaire (good mood, alertness and calmness), whereas the improvement reached statistical significance only in the subcategory of alertness for the scrotal gel group. The results at week 24 were not significantly different between the three treatment groups.

With regard to the total score of the Derogatis Questionnaire, there were also no significant differences between the three groups or obvious trends during the course of the study. However, focusing on the subcategories, there was a significantly positive trend in all three treatment groups related to orgasm and sexual drive/relationship. Both sexual arousal and sexual behaviour/experience showed a significantly positive trend in the dermal gel and the latter also in the patch group.
When we analysed patients under 60 years of age $(n=110)$, we also found a significant improvement in sexual cognition/fantasy in the dermal gel group. In the subcategory of sexual arousal, there was a significantly higher median score ( \pm IQ range) at weeks 20 and 24 in the dermal group $(17.5 \pm 7.5)$ than the patch $(14 \pm 11)$ or the scrotal gel group $(13 \pm 10.5)$. IPSS score as well as quality of life score did not show noticeable differences between treatment groups, and no temporal trends of change were observed in the course of the study.

\section{Tolerability, adverse events and dropouts}

Tolerability was significantly better in both gel groups than the patch group at every visit, judged by patients and physicians $(P<0.01)$. In the gel groups, tolerability was assessed as 'very good' or 'good 'in more than $92 \%$ of the patients, but in only $53.5 \%$ in the patch group. At weeks 20 and 24, none of the patients in the gel groups assessed tolerability as 'very poor' or 'poor', in contrast to $14-16 \%$ of the TTP group. The incidence of adverse events and severe adverse events not causally
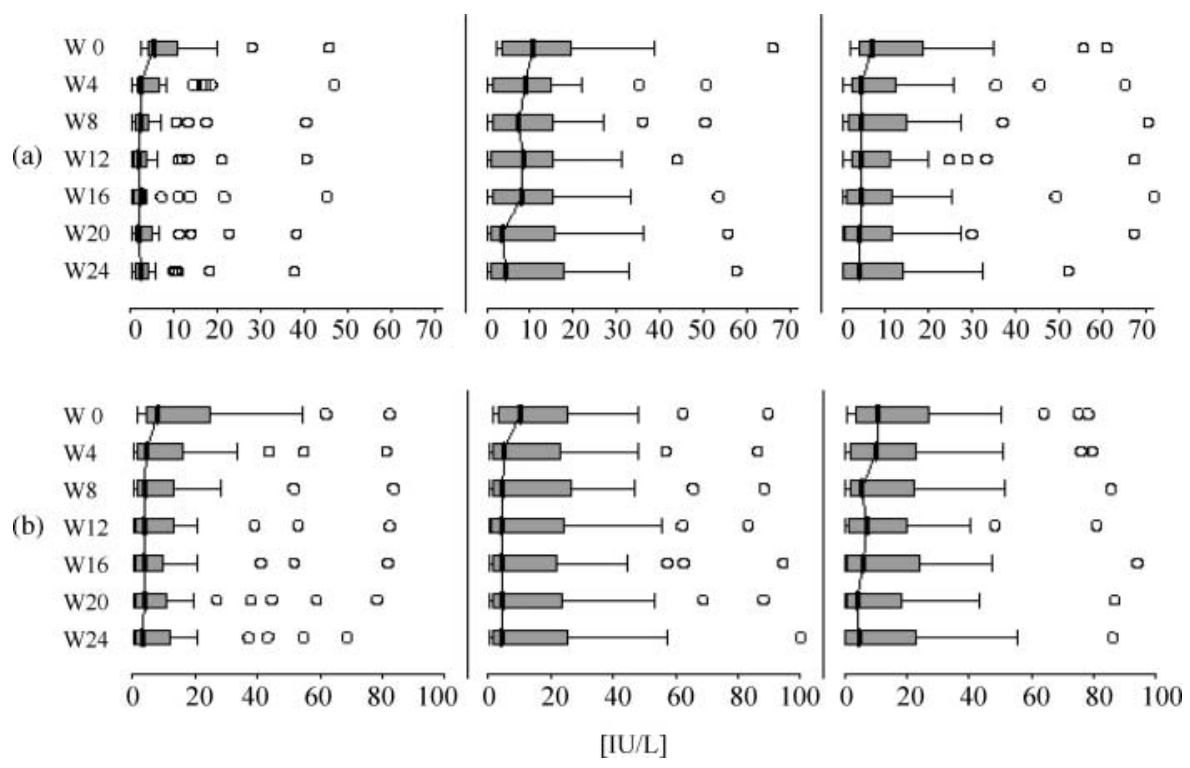

Figure $6 \mathrm{LH}$ (a) and FSH (b) levels in the patch, scrotal and dermal groups at weeks $0-24$ (W00-24). 
(a)
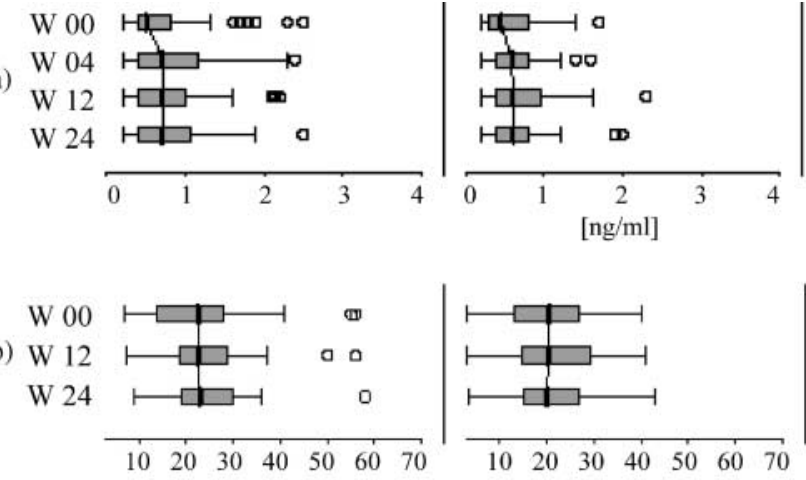

[ml]

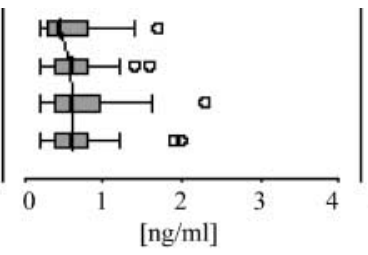

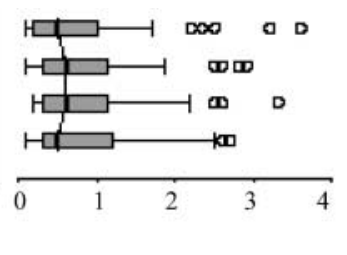

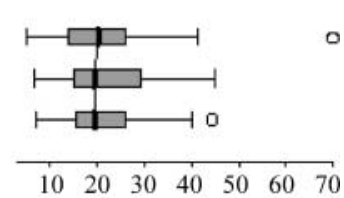

Figure 7 PSA levels (a) and prostate volume (b) in the patch, scrotal and dermal groups at weeks 0-24 (W00-24). related to the medication did not differ significantly between groups.

Significantly more skin reactions at the administration site were observed in the TTP group (13 patients) than in the scrotal or the dermal groups (two patients respectively) (one-sided U test; $P<0.01$ ). Six patients of the TTP group dropped out due to skin reactions, while no patient dropped out in either of the other groups for this reason. In the case of the patch application site, irritant reactions, erythema or pruritus were described. In the scrotal gel group, one patient also complained of pruritus after gel application and another of burning at the application site. One patient in the dermal group reported dryness at the application site, and another observed erythema when he did not remove the gel and perspired at the same time. These reactions disappeared spontaneously.

\section{Discussion}

For testosterone gels clinically available to date, an absorption rate of about $10 \%$ is assumed so that a large volume of gel remains on the surface of the skin after application $(8,9)$. Even $12 \mathrm{~h}$ after application of one of these gels by the male subject $(8,9)$, a significant increase in testosterone levels in women was demonstrated when the couples had vigorous skin contact. The increase was the same when the rubbing experiments were performed $4 \mathrm{~h}$ after gel application and the women showered directly after the rubbing activity (9). Consequently, after application of these gels, patients are requested to avoid skin contact with partners or children or to cover the application sites with clothing after the gel has dried to prevent interpersonal transfer. They should also avoid showering or swimming for several hours to guarantee the assumed resorption. For TGW, the gel analysed in this study, it was previously shown that $49.7 \%$ of the gel remains on the skin up to $8 \mathrm{~h}$ after application. The amount is reduced to $9.1 \%$ when the skin is washed and dried after application. The same study showed no risk of interpersonal transfer after removal of the gel (6). Simultaneously, it was also shown that intensive skin contact 10 min after application does not increase testosterone levels of the recipient, even without a washing procedure. After $10 \mathrm{~min}$, the alcohol, a gel ingredient serving as enhancer for transdermal permeation, evaporates. Preliminary studies had demonstrated that the removal of TGW 10 min after application resulted in sufficient testosterone serum levels in gonadotrophin-suppressed normal men after scrotal or non-scrotal application (6).

Other gel studies cited above come to different conclusions about the risk of transfer, as 1. they analysed the transfer to women and not to gonadotropinsuppressed men; 2. skin contact was 5 min longer than in the previous TGW study; or 3. the composition of the gels causes different resorption rates. However, as considerable amounts of gel remain on the skin and could be transferred to and theoretically absorbed by another person, its removal by washing would further minimise the risk of transfer. For approved gels, efficacy and safety have not as yet been tested in respect of short-term administration of the gel followed by its removal.

In the present study, the dermal gel group proved to be not only equivalent to the patch group at week 24 but even better. This was true for free and total testosterone as well as combined testosterone and DHT, and in different study populations (ITT and PP). The scrotal gel group was equivalent to the patch group with regard to testosterone and when the combined levels of testosterone and DHT were analysed, it proved to be more effective than the patch group in the test of equivalence. As the patches were applied in the evening, according to the instructions of the company, the kinetics of this group do not include the first few hours after application. Consequently, the $t_{\max }$ values differ from other reports, that is, $8.1 \mathrm{~h}$ vs. $12 \mathrm{~h}$ in our study, and the hormone parameters may have been underestimated. This is a limiting factor in our study design, when kinetic parameters of the different treatment groups are compared. 
There are, however, several more important clinical parameters to compare efficacy. In the Mood Disorder Questionnaire, the Quality of Life score and the Derogatis Questionnaire, there were no significant differences between the three treatment groups, indicating that the gel is as efficient as the patch. When a subgroup analysis was performed and only patients under 60 years of age were included, the dermal group performed even better in the subcategory of sexual arousal. The trends for improvement reflected in both the Mood Disorder Questionnaire and the Derogatis Questionnaire were small, and this might be related to a high percentage of pretreated patients and a relative short wash-out period, compared with other studies. Swerdloff et al. (10) had a withdrawal period of 6 weeks after injection therapy and 4 weeks with oral or transdermal treatment.

TGW is as safe as the patch, which was supported by PSA levels, prostate volumes, IPSS, haematology and clinical chemistry. However, significant differences between the groups were observed with regard to tolerability. The poor tolerability of the patch is caused by a high rate of skin reactions, which is also reflected by a high dropout rate in this group. In contrast, none of the patients in the gel groups complained about persisting skin reactions or discontinued the study because of skin reactions.

This is the first study dealing with application of a testosterone-containing gel to the scrotum. Because of the fivefold higher resorption rate of the scrotum, we used a fivefold lower dose than in the non-scrotal arm. Clinically, patients were well adjusted despite this low dose, which is reflected by the very low rate of drug adjustment. In contrast to the scrotal patches available formerly, clipping scrotal hair is not necessary when using the scrotal gel.

As there is a high concentration of $5 \alpha$-reductase in scrotal skin, we determined DHT kinetics in the scrotal gel group to assess the full androgen spectrum. DHT levels at week 24, analysed before gel application in the morning, were in the supraphysiological range in both the dermal and the scrotal gel group, with a higher increase in the scrotal group. The fivefold increase in the dermal group is comparable to that found in a pharmacokinetic analysis of $100 \mathrm{mg}$ AndroGel applied to non-scrotal skin (10). Surprisingly, we found similar DHT levels in the dermal and scrotal gel group at week 24 when we analysed the levels $2 \mathrm{~h}$ after gel application. Other gel studies have also shown that DHT levels increase more after gel application than after application of the (non-scrotal) patch. This may be caused by the fact that $5 \alpha$-reductase is also detectable in all skin types, and the larger the area covered by the gel, the higher the conversion rate of testosterone to DHT.

As DHT is implicated in the development of $\mathrm{BPH}$ and prostate cancer, high DHT blood levels might be regarded undesirable. Clinical studies with scrotal testosterone patches provide a long-term outcome with high DHT blood levels over a period of several years that do not indicate any risk of prostatic hyperplasia or cancer, although they involve only a small number of patients (11). Furthermore, three DHT gel studies did not report any increase in PSA levels, prostate volume or symptoms $(12-14)$. However, these are short-term studies over 3 or 6 months. In one study, even a $15 \%$ decrease of prostate volume was detected (15), and this is possibly due to the following mechanism: DHT suppresses LH and FSH levels and thereby also testosterone and the aromatisation of testosterone to estradiol, which is involved in the development of benign prostatic hyperplasia (BPH) (15). Additionally, an endocrine autonomy of the prostate (16), resulting in higher intraprostatic DHT levels than serum DHT levels and higher intraprostatic DHT levels than intraprostatic testosterone levels, may be responsible for the missing growth effect of external DHT on the prostate. In the light of these data, we do not see a cause for concern in respect of the elevated DHT serum levels detected in this study. Nevertheless, a tight follow-up of PSA levels and digital rectal examination of prostates in patients treated with the testosterone gel remains mandatory (17).

DHT has been suggested as sole treatment for hypogonadism (12-14). In contrast to this monotherapy, testosterone gel has the advantage that not only DHT but also testosterone and estradiol, another testosterone metabolite, are elevated in blood. Normal estrogen levels are a prerequisite for maintaining normal bone density.

In summary, the study shows that the scrotal application of TGW is as effective and safe as the patch, whereas dermal application of TGW is superior to the patch and scrotal gel application. Both modes of gel application guarantee better tolerability than the patch and, in comparison to other gels on the market, provide higher flexibility and lower chance of interpersonal transfer, due to the early removal of the gel.

\section{Acknowledgements}

The Wolff Multi-Centre Study Group includes: H-U Eickenberg, Franziskus Hospital Bielefeld; J Brüning, University of Cologne; G Haidl, T Hagemann, University of Bonn; F Dessel, A Matuszczyk, K Mann, University of Essen; H M Behre, University of Halle; J Jakobeit, H M Schulte, Endokrinologikum Hamburg; E Leifke, G Brabant, University of Hannover; A Kastein, S Lenk, University of Berlin; A Krisp, W Krause, University of Marburg; C Hagel, urologist in Nürnberg; K Reschke, H Lehnert, University of Magdeburg; U Van der Haegen, K-H Usadel, University of Frankfurt/Main; H J Glander, U Paasch, H Janus, University of Leipzig; A Recker, H-C Schuppe, W-B Schill, University of Giessen. The technical assistance of S. Müller-Röhr (Wolff, Bielefeld), S Borchert and J Esselmann (Institute of Reproductive Medicine, Münster) is gratefully 
acknowledged as well as language editing of this manuscript by S. Nieschlag MA. The study was supported by Dr Wolff GmbH, Bielefeld, Germany.

\section{References}

1 McNicholas TA, Dean JD, Mulder H, Carnegie C \& Jones NA. A novel testosterone gel formulation normalizes androgen levels in hypogonadal men, with improvements in body composition and sexual function. British Journal of Urology International 200391 $69-74$.

2 Steidle C, Schwartz S, Jacoby K, Sebree T, Smith T \& Bachand R. North American AA2500 T Gel Study Group. AA2500 testosterone gel normalizes androgen levels in aging males with improvements in body composition and sexual function. Journal of Clinical Endocrinology and Metabolism $2003 \mathbf{8 8} 2673-2681$.

3 Wang C, Cunningham G, Dobs A, Iranmanesh A, Matsumoto AM, Snyder PJ. Weber T, Berman N, Hull L \& Swerdloff RS. Long-term testosterone gel (AndroGel) treatment maintains beneficial effects on sexual function and mood, lean and fat mass, and bone mineral density in hypogonadal men. Journal of Clinical Endocrinology and Metabolism 200489 2085-2098.

4 Jordan WP Jr. Allergy and topical irritation associated with transdermal testosterone administration: a comparison of scrotal and nonscrotal transdermal systems. American Journal of Contact Dermatitis $19978108-113$.

5 Rolf C, Knie U, Lemmnitz G \& Nieschlag E. Interpersonal transfer after topical application of a newly developed testosterone gel preparation. Clinical Endocrinology (Oxford) 200256 637-641.

6 Rolf C, Kemper S, Lemmnitz G, Eickenberg U \& Nieschlag E. Pharmacokinetics of a new transdermal testosterone gel in gonadotrophin-suppressed normal men. European Journal of Endocrinology $2002146673-679$.

7 International Conference on Harmonization of Technical Requirements for Registration of Pharmaceuticals for Human Use (ICH): Good Clinical Practice: Consolidated Guideline; 30 April 1997.

8 Unimed-Pharmaceuticals Inc.; A Solvay Pharmaceuticals, Inc. Company. AndroGel® prescribing information for healthcare professionals. Solvay Pharmaceuticals, Inc., Marietta, GA, USA 2004.
9 Auxilium Pharmaceuticals, Inc. Testim ${ }^{\circledR}$ prescribing information for healthcare professionals. Auxilium Pharmaceuticals, Norristown, PA, USA 2004.

10 Swerdloff RS, Wang C, Cunningham G, Dobs A, Iranmanesh A, Matsumoto AM, Snyder PJ, Weber T, Longstreth J, Berman N and the Testosterone Gel Study Group. Long-term pharmacokinetics of transdermal testosterone gel in hypogonadal men. Journal of Clinical Endocrinology and Metabolism 200085 4500-4510.

11 Behre HM, von Eckardstein S, Kliesch S \& Nieschlag E. Long-term substitution therapy of hypogonadal men with transscrotal testosterone over 7-10 years. Clinical Endocrinology 199950 629-635.

12 Ly LP, Jimenez M, Zhuang TN, Celermajer DS, Conway AJ \& Handelsman DJ. A double-blind, placebo-controlled, randomized clinical trial of transdermal dihydrotestosterone gel on muscular strength, mobility, and quality of life in older men with partial androgen deficiency. Journal of Clinical Endocrinology and Metabolism $2001864078-4088$.

13 Kunelius P, Lukkarinen O, Hannuksela ML, Itkonen O \& Tapanainen JS. The effects of transdermal dihydrotestosterone in the aging male: a prospective, randomized, double blind study. Journal of Clinical Endocrinology and Metabolism 200287 1467-1472.

14 De Lignieres B. Transdermal dihydrotestosterone treatment of 'andropause'. Annals of Medicine 199325 235-241.

15 Wang C \& Swerdloff RS. Editorial: Should the nonaromatizable androgen dihydrotestosterone be considered as an alternative to testosterone in the treatment of the andropause? Journal of Clinical Endocrinology and Metabolism $2002871462-1466$.

16 Krieg M. Biochemical endocrinology of human prostatic tumors. Progress in Cancer Research Therapy 198431 425-440.

17 Nieschlag E, Swerdloff R, Behre HM, Gooren LJ, Kaufman JM, Legros JJ, Lonenfeld B, Morley JE, Schulman C, Wang C, Weidner $\mathrm{W} \& \mathrm{Wu}$ FC. Investigation, treatment and monitoring of late onset hypogonadism in males: ISA, ISSAM, and EAU recommendations. International Journal of Andrology $2005 \mathbf{2 8}$ $125-127$.

Received 31 March 2005

Accepted 13 May 2005 